\title{
Federated Accounting: Service Charging and Billing in a Business-to-Business Environment
}

\author{
B. Bhushan \\ M. Tschichholz \\ GMD-FOKUS \\ Kaiserin-Augusta-Allee 31 \\ D-10589 Berlin Germany \\ Tel : +49-30-3463 7351 (3463 7215) \\ \{bhushan,tschichholz\}@fokus.gmd.de
}

\author{
E. Leray \\ W. Donnelly \\ Telecommunications Software \\ Systems Group \\ Waterford Institute of Technology, \\ Ireland \\ Tel : +353-51-302412 (302423) \\ \{eleray,wdonnelly\}@tssg.wit.ie
}

\begin{abstract}
The liberalisation of the telecommunications industry has resulted in a proliferation of new services and services providers. This is particularly the case in the rapidly expanding IP-based services market. Providers include Internet Service Providers (ISPs), Virtual Private Network (VPN), communication (backbone operators), and application service providers. The final service set delivered to the customer enduser will result from the combination of these service offerings. From a service usage accounting perspective, this new environment creates a number of important challenges, which did not apply in the traditional monopolistic telecommunications environment. The first issue is that of multiple administrative domains from the customer and service provider side and the second issue concerns applying customer Service Level Agreements (SLA) and Quality of Service (QoS). This paper addresses the issue of settlement of service usage charging across several service providers when they collectively provide application, information, or communication service to a customer. The issue at the stake is to develop standardised mechanisms that allow various business and operation support systems operating in various domains to exchange service usage information.

The work presented in this paper focuses on the development of a business-tobusiness (B2B) service provisioning and management architecture to provide guidance to international organisations on the development of a federated accounting management solution. This architecture aims to be both open and adaptable and draws requirements from standardisation work going on in TMForum (TeleManagement Forum), IPDR (IP Detail Record), IETF (Internet Engineering Task Force) and ETSI (European Telecommunication Standardisation Institute).
\end{abstract}

\section{Keywords}

Business-to-business environment, service usage charging and billing, federated accounting management, SLA and QoS provisioning. 


\section{Introduction}

Over the last 5 years the telecommunications industry structure has changed dramatically. Deregulation has resulted in the opening the telecommunications markets worldwide. The nature of telecommunication networking has also changed because the traffic started to move away from traditional circuit-switched network onto a rather un-structured IP backbone [1]. These major changes have brought to the surface a rapidly growing worldwide B2B e-commerce market. From an estimated B2B market range of $\$ 2$ billion to $\$ 3$ billion in 1998 [2] present figures project $\$ 2.7$ trillion worth of e-commerce transactions for 2005 [3]. The changes in the telecommunications market and the emergence of the B2B market are having a major impact on the way the industry understands and approaches service billing.

The main impact of $\mathrm{B} 2 \mathrm{~B}$ on service billing is due to the complexity of the $\mathrm{B} 2 \mathrm{~B}$ business model and the customers' expectations from services. In a B2B environment, customers have is a complex business structures and their expectation toward billing in terms of on-line services and customer care are higher. Hence, the demands and needs of such customers have already changed in terms of what is billed and how it is billed. B2B Billing systems must be capable of generating a service bill which integrates the charges for the various subservices while at the same time integrating any discounts or costs in line with the Customer Service Level agreements. For instance charging for a customer service such as Voice over IP (VoIP) may require the integration of ISP, connectivity and VoIP Application provider charges as well as any discounts due to customer QoS requirements as defined in the customer SLA. Legacy billing systems owned or outsourced by Service Providers (SP) will not be able to cope with the complex billing methodologies for these next-generation network services. It is acknowledged widely that it is through its billing system that a business communicates with its customers. Furthermore, optimising billing is a matter of critical importance for gaining a competitive advantage that, in the telecommunications industry, has become a vital parameter for Service Provider survival.

The IST (Information Societies Technology) project FORM ${ }^{1}$ aims to provide a development framework for the management of this new B2B environment. FORM's main objective is to engineer an open, adaptable framework to support the Management of an Inter-Enterprise Service encompassing telecommunication facilities and systems by an external provider. A primary concern in FORM is that such an Inter-Enterprise solution will not take off if it cannot be accounted valuably. Hence, FORM accounting and billing management research area includes:

- Multi-domain accounting and billing solution in a federated service provider environment;

- Management solution for equitable cost allocation and revenue (for sharing accounting information, generating revenues and auditing revenue generation);

- Management solution for differentiated charging, usage-based, applicationspecific accounting and billing.

\footnotetext{
FORM project full title "Engineering a Co-operative Inter-Enterprise Management
} Framework - Supporting Dynamic Federated ORganisations Management" 
The objectives of the paper are twofold: firstly, to identify the principle emerging issues of service accounting in a Business-to-Business environment; and secondly, to present an architectural framework for the development of federated accounting management systems for such an environment. The paper concludes by reporting the progress of the research work done so far in the FORM project.

\section{Service Usage Accounting Issues in a Business-to-business Service Provisioning Environment}

From the point of view of service usage billing, the IP-based networks pose a number of challenges of which service usage collection in a multi-domain environment and SLA and QoS management are the most important.

\subsection{Service Usage Data Exchange in an Multi-domain Environment}

The IETF group defines Inter-domain accounting as "an accounting process that involves the collection of information on resource usage of an entity with an administrative domain, for use within another administrative domain" [4]. Hence, in inter-domain accounting, accounting packets and session records will typically crossadministrative boundaries. In a multi-domain environment there are often different accounting systems employed and therefore different ways of representing accounting information are likely to exist in each domain. Thus, accounting packets and session records crossing boundaries is not a trivial problem.

In a B2B environment, the majority of services are operated across a multidomain environment and hence B2B accounting systems must support inter-domain accounting. Thus the capture and exchange of usage information for billing purpose is fundamental. In such an environment accounting packet loss, in an inter-domain transaction, may translate directly into revenue loss.

\subsection{Service Level Agreement (SLA) and QoS Management}

Another issue raised by the deployment of next-generation IP services is that best effort service is not good enough for B2B customers, who tend to have a higher level of expectations from commitments they negotiate with third party service providers. These commitments are expressed as a SLA. SLAs are about a customer and a service provider agreeing on both the types of services to be provided and the level of quality at which those services are to be provided. ISPs are therefore challenged to provide and charge for services that must meet quantifiable commitments.

The key issue in this context is the mapping between these agreements (SLA) and the QoS reported, and the final impact it has on a customer bill. This is not a trivial problem, as various players from diverse domains (ASP, ISP and other SP) need to agree on some basic principles if they want to deliver and measure these new services consistently. Therefore the key challenge here is to develop interfaces, by which various support systems can exchange usage, provisioning and control information with each other.

In summary, the IP-based service industry sector has now recognised the need for a common method of defining usage data transactions, without which usage-based billing and charge settlement between IP operators will be impractical [5]. Accounting in federated service provisioning environment is an upcoming topic. 
It is important to base federated accounting solutions on widely used open standards and tools to gain acceptance in a broad user community.

\section{Technological Context}

This section places the idea of federated accounting management in the context of the FORM project and existing technology. The idea of InterEnterprise Service (IES) and IES Provider (or IESP) form the basis of the federated accounting concept. The IESs comprise of management services supporting dynamic federated organisations performing businesses across IP based Intranet, Extranet or Internet. Examples are SMEs (Small and Medium Scale Enterprise) participating in B2B ecommerce or application service providers (ASPs) and their customers. B2B itself is defined as commerce conducted between businesses over an Intranet, Extranet or Internet (i.e. IP networks). This trade may be conducted between businesses and its supply chain as well as between a business and other business end-customers. As illustrated in the figure 1, requirements for federated accounting management are derived from previous European research projects (FlowThru, Bandwidth2000) and organisations that are at the forefront of the charging and billing system specification (IETF, TMF etc). In order to derive and classifying functional requirements, TMForum's TIM (Technological Integration Map) [6] and TOM (Telecom Operation Maps) [7] are used. There are four main classes of functional requirements: static functionality, dynamic functionality, abnormal conditions, and administrative requirements, which are summarised in section 3.3.

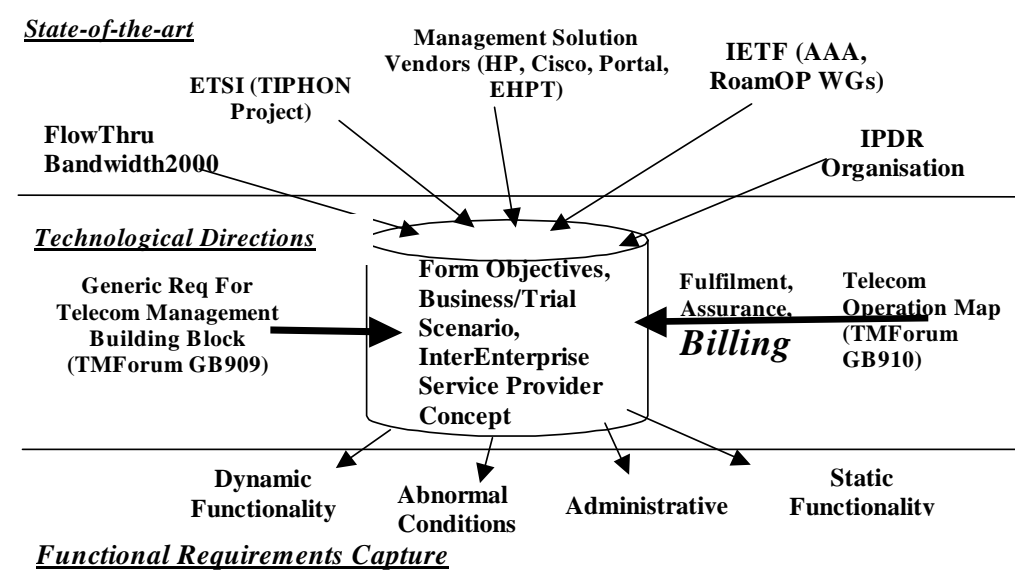

Figure 1: Technological Context for Federated Accounting Management within FORM Project.

\subsection{State of the Art}

This section briefly reports on the state-of-the-art in charging and billing within industrial and international organsations.

Developing usage accounting solution for advanced IP services is a major challenge. One of the main providers of accounting and billing services for IP based 
networks are the vendors of equipment, mediation and billing products. The most important vendors are HP, EHPT, Cisco, and Portal. The solutions allow ISPs and Telcos to develop and operate operations and business support systems. However these solutions tend to be proprietary. Currently, organisations such as IPDR, ETSI's TIPHON (Telecommunications and Internet Protocol Harmonisation Over Networks) projects, IETF AAA WG (Authentication, Authorisation and Accounting Working Group), and TMForum have begun to develop more standardised accoutning solutions for the IP services The most important areas for standardisation is the settlement of service usage charging accounts among several service providers (or to be precise, the exchange of usage and accounting information across the boundaries of service providers' domains). The AAA WG acknowledges that the application services, which are in use and being developed, are divers and therefore managing them will require various a set of standardised accounting management protocol and services. [8], [9].

The purpose of IPDR organisation is to define the essential elements of data exchange between network elements, OSS (Operation Support System) and BSS (Business Support System). The definition will provide the foundation for development of open, carrier-grade IP support systems that will enable nextgeneration IP networks to operate efficiently and cost effectively. For the specification of interfaces between OSSs and BSSs, the IPDR organisation has adopted the core functional roles and interfaces that TMF's Telecommunications Operation Map (TOM) [7] has identified. The main goals of IPDR organisation are [10] to define an open, flexible record format (the IPDR record) for exchanging usage information and essential parameters for any IP transaction.

The objective of TIPHON project is to standardize voice communication (e.g., telephony) over the Internet. As part of overall TIPHON specifications, ETSI is also working in the area of requirements for service interoperability, technical aspects of charging/billing and security [11], [12].

The purpose of the Bandwidth 2000 project was to facilitate the introduction of on-line accounting, ordering, billing, and brokering of Internet services that support telecom market place [13]. The goal of FlowThru ${ }^{2}$ was to provide the industry with a concrete guidance for building an optimum solution to specific management problems from the wide range of architectural and technological approaches that were available from bodies such as the ITU-T, ISO, TMForum, TINA-C, OMG, ETSI and EURESCOM [14].

\section{Analysis}

Frameworks and recommendations (a prime example of which is TMN, or Telecommunication Management Network) that have been produced by telecommunications standardisation work have allowed telecommunications industry to categorise management functions by logical layers (business, service, network and element) as well as functional areas (fault, configuration, accounting, performance and security). However, IP-based networks do not benefit from any such functional architecture and there is a lack of a concerted effort toward methodologically

\footnotetext{
${ }^{2}$ FlowThru (ACTS program under contract number AC335, full title "Co-operative secure management of multi technology and administrative domain Network and Service Management Systems").
} 
applying the TMN framework to IP-based networks. The projects mentioned in this section have taken strides towards applying the TMN and TMForum frameworks to IP-based networks. There are still outstanding issues that need to be addressed in the light of B2B service provisioning and usage environment. In this rapidly growing environment autonomous enterprises set up inter-enterprise relationships and outsource their communication and management needs to a business-to-business communication service provider (that is, inter-enterprise service provider). Service provisioning and charging and billing management must be augmented with tools that take care of the concerns of QoS assurance and SLA handling, which tend to be high in B2B environment. Thus, it is important to develop an open and standardised framework that allow various BSSs and OSSs operating in autonomous domains to exchange service usage and charging information. The research work presented in this paper benefits from the results of standardisation task going on in TMForum, IPDR, IETF and ETSI in order to tackle this issue. Most importantly, TMForum's core charging and billing functional roles [TOM-GB910], and IPDR organisation's specification [10] and IPDR XML Schema are used. The initiative in the area of federated accounting aims to contribute to IPDR and TMForum specifications by providing the two organisations with the result of implementations of their specifications.

\subsection{Functional and Technological Direction}

As illustrated in Figure 2, the charging and billing business process that this paper targets is described in the context of the FAB (Fulfilment, Assurance, and Billing) of TOM (Telecom Operation Map) [7]. IPDR XML Schema [10] is used to model and specify elements of accounting information that are exchanged between various business processes, operating in IESP and third-party SPs domains.

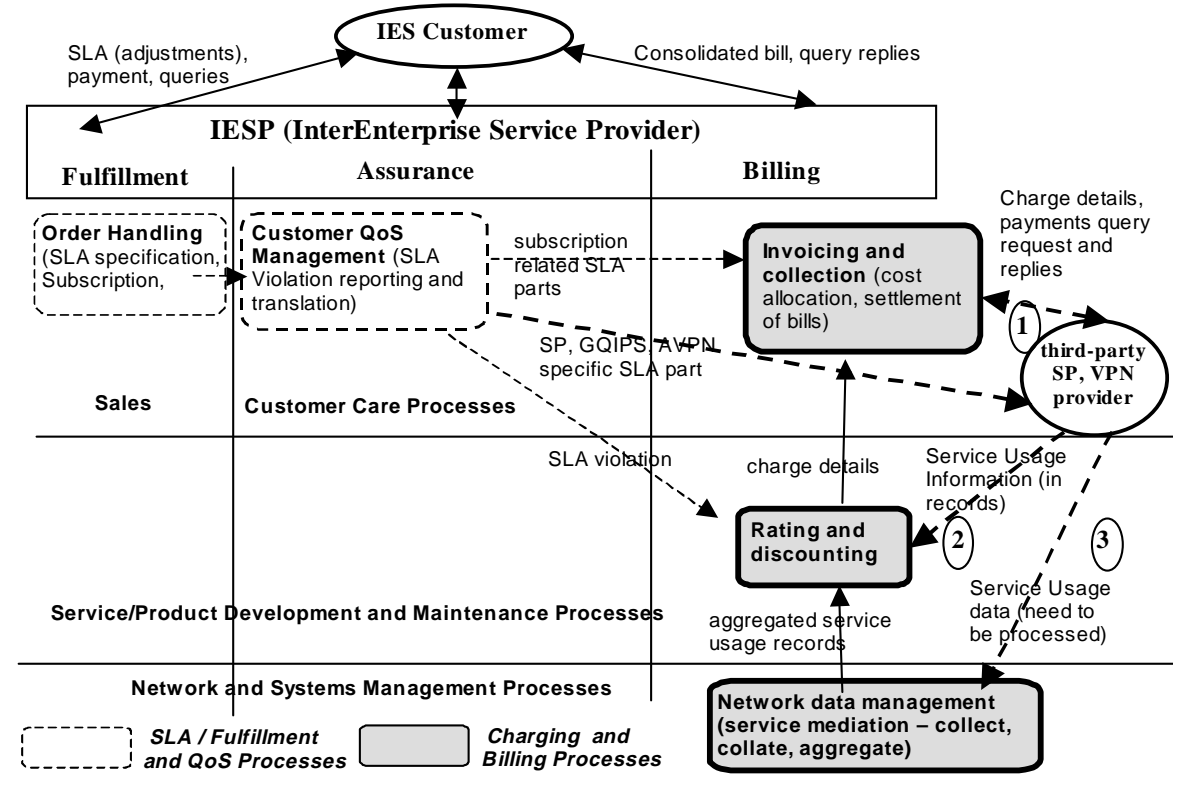

Figure 2: Charging and Billing Business Processes. 
Figure 2 also illustrates a scenario where an Inter-Enterprise Service Provider (IESP) manages the outsourced B2B services for a customer. These services are provided by a number of Third party Service Providers (third party SP) and a VPN provider. The role of the IESP is to monitor the service usage and produce an integrated service bill to the customer. The charging and billing business processes are divided into three sub-business processes. The functionality of each sub-process is dependent on OSS third-party SPs use. The third-party SP and VPN provider can use at least three types of mediation, charging and billing management service from the IESP, depending on type of OSS. The three types of accounting management service are described below.

\section{Consolidated Billing (Dotted arrow 1 in figure 2)}

VPN and third-party SP use OSSs to do full-fledged processing of charge for the services that customers use. They forward details of the charges (or bills contents) to IESP, who consolidates bills, allocates the charges, and returns the payments. The accounting information, e.g., the details of service usage and charges, that is exchanged among IESP, and VPN and third-party SP is specified in form of XML Document.

Rating, Discounting, and Consolidated Billing (Dotted arrow 2 in figure 2) VPN and third-party SP use mediation OSSs to produce service usage information in form of IPDR documents and forward their service-specific IPDR documents to IESP, who processes the document with rating and discounting management processes. Consolidated bills are sent to customers and payments are returned to VPN provider and third-party SP. Information exchanged between VPN provider and third-party SP, and IESP are service usage records (SDRs) in a standardised format.

Service Mediation, Rating and Consolidated Billing (Dotted arrow 3 in figure 2) VPN and third-party SP use OSSs, which do very little information processing (e.g., collecting usage data from application servers), and the rest of mediation, charging and billing task is left to IESP. IESP does the service mediation and produces service usage information in exchangeable record format (e.g., XML). Then, rates and discounts are applied and the final consolidated bills are prepared. Consolidated bills are sent to customers and payments are returned to VPN provider and third-party SP. Information exchanged between IESP, VPN provider and third-party SP is service usage data, in the form that is typically fed into the service mediation process.

Having studied the state-of-the-art and taken directions from TMForum, the following subsection summarises the main functional requirements for federated accounting.

\subsection{Functional Requirements}

The functional requirements for the federated accounting system are mainly derived on the basis of the TMF TIM (Generic Requirement for Telecommunication Management Building Block) framework [6]. The following summarises the main requirements.

\section{Static Functionality Requirements}

Usage Information: Accuracy of service usage data must be maintained while exchanging usage data at all interfaces. For detailed charging and cost allocation, 
granularity of usage data must be high so that accurate information can be extracted from service usage records.

Specification or requirement and definition of interfaces (or contracts, as they are termed in [6]: The interfaces must be specified in a standardised specification language. Developer must be able to trace back the source of contracts to use case and component diagrams that are created using UML notations.

\section{Dynamic Functionality Requirements}

Combination of Charging Schemes and tariffs: An IESP must be able to apply a combination of two or more charging schemes and tariffs. Customer can be charged at a flat rate but pricing a service is determined by the network technology that a customer uses to access the service. Customer can be charged at flat rate but only to a limited usage period. Any usage that goes over a limit can be charged at a rate. Therefore service provider should be able to ascertain: (1) The features of a service that are prone to be over-used; (2) The manner in which they over-used; and (3) The time when they can be over-used. An IESP should also be able to charge different services at different rates on the basis of usage. Service charges may be calculated on the basis of the features of services and customer needs.

Exchangeable Usage Record Format: In order to support charging and billing in an environment in which service provider form a federation and operate, the IESP must be able to make use of usage record format that is exchangeable. The following requirements must be met: (1) syntax and semantic of exchangeable record format; (2) translation mechanism for converting usage record from a service-specific format into one which is exchangeable; (3) consistency of the exchangeable service usage record format; and (4) availability of service usage record in an exchangeable format from all service providers who operate in a federated environment.

SLA between IESP and customer: SLA at this level should specify the terms and conditions of the application service that IESP is to provide and customer is to receive. In a SLA, customers and IESP agree on the level of QoS that IESPs are to maintain. Customers expect to receive that level of QoS through out the period during which they use services.

SLA between IESP and a third-party service provider: SLA at this level should specify the terms and conditions of the charging and billing service that IESP is to provide to VPN provider and ASP.

\section{Abnormal Conditions}

Detection of Unusual Usage Session: Unusual usage sessions must be detected. If users need to use a service in an unbounded session, access to services should depend on quotas and credits they have.

Prevention of Service Overuse: The billing services are expected to meet the requirements of financial and legal regulations of an organisation. If charges of a service usage are high and run up quickly, it is essential to have real-time accounting to prevent users from overusing the service. Usage accounting should be completed within a defined time window.

Limiting Service Usage: IESP should be able to limit service usage. For example, only customers are allowed to use a service that is not available for free and the number of customers who have access to the application or service can also be Limited. Similarly, a limited number of customers should be allowed to connect to a service simultaneously. 


\section{Administrative}

The system must be extensible and must allow an administrator to extend the functionality to measure the usage of new services. It must be possible to define service-specific extensions and new standard-specific and vendor-specific attributes.

\section{Architectural Framework}

Figure 3 illustrates the model of the FORM billing business process. The main players are IES Customers, an IES Provider (IESP), a VPN Provider, a GQIPS (Guaranteed QoS IP Service) provider, and a third-party SP.

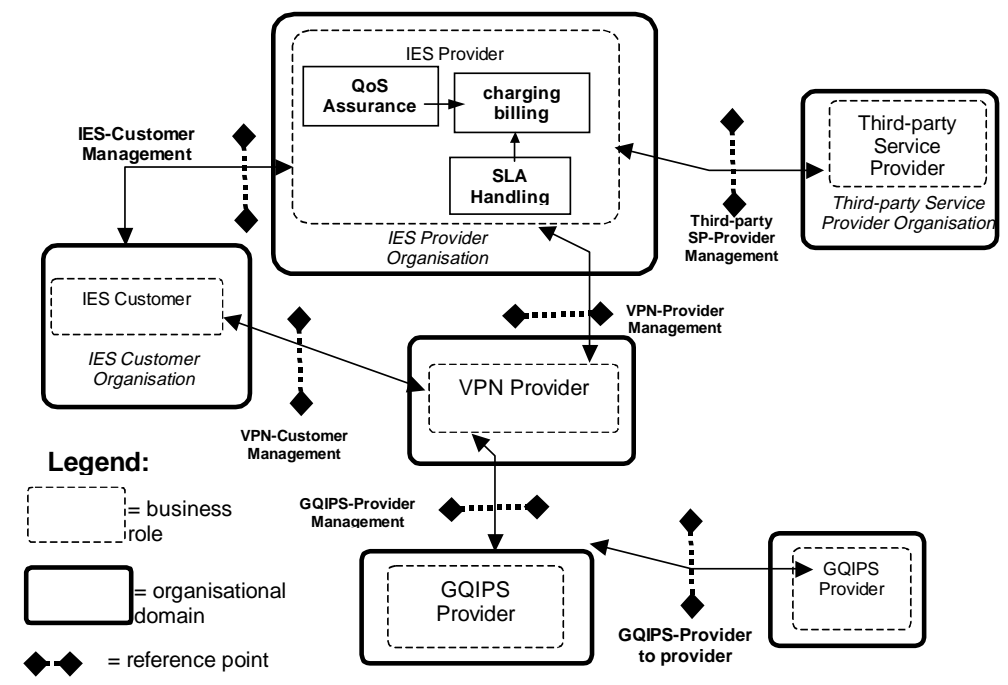

Figure 3: Business Model for the Federated Accounting.

From the point of view of accounting and billing functionality, it must be noted that IESP, VPN Provider, GQISP, and third-party SP may operate OSSs to carry out a range of tasks (from service negotiation and aggregated usage record generation, to full-fledged billing).

IES customer receives bills from IESP, and this bill includes the charges incurred from using application service. In this case, the role of IESP will be fourfold: (1) negotiate between third-party SP and the customer; (2) maintain customer SLA; (3) settle the charges amongst third-party SPs, VPN Provider, GQIPS, etc., for the services that they provide; and (4) bills the customer. If two third-party SPs provide services, the IESP will also merge bills into a consolidated bill and forward consolidated bill to IES customer.

Third-party SP and VPN Provider may have their own OSSs and the functionality of such OSSs may range from service mediation to full-fledged charge processing. Hence, there can be various ways by which third-party SP and IESPs can co-operate. It will depend on many aspects such as level of detail of information they can exchange, inter-operable interfaces, and type of B2B relationship between thirdparty SP and IESP (specified in SLA). 
The federated accounting in FORM is based on the idea of inter-domain accounting in which two domains exchange SDRs (Service Detail Records) in form of XML-based IPDR documents for the settlement of charges. For example, an IESP (with its own domain) and third-party SPs (with their own accounting systems) settle service charges by exchanging accounting information. Accounting information can be service-specific usage data in IPDR document (see information model section 4.3, which illustrates an information model of Media-on-demand (an application service similar to Video-on-demand). Or, it can be an extended IPDR document that contains service-specific usage data as well as charge information (more discussion are given in the section 4.2 with table 1 showing what information this extended IPDR document may contain).

This form of inter-domain accounting can be considered federated accounting, where federated accounting depends on the definition of the interoperable interfaces and accounting information to be exchanged between IESP and third-party SP.

\subsection{Business Scenario (VoIP/VPN multi-domain accounting)}

The external actors who interact with the federated accounting management define the boundary of the Federated Accounting architecture. These actors and the roles that they play in the charging and billing management scenario are illustrated in figure 4 and described below:

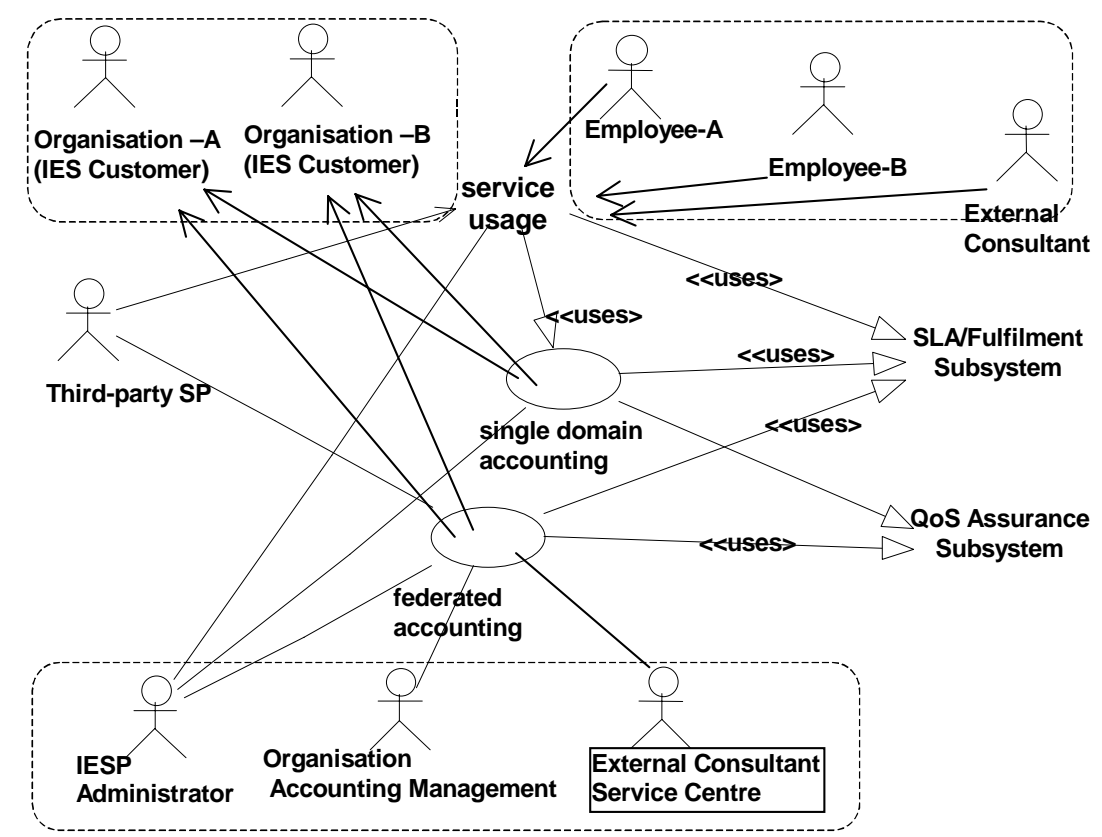

Figure 4: A Boundary of Federated Accounting Management System.

In a typical service usage and charging scenario employees of two organisations (Organisation-A and Organisation-B), which are IES Customers, want to use a communication service (e.g., Voice or Fax over IP) in order to communicate with 
each other. Employees of the two organisations may also at some point in time seek expert advice from an External Consultant and at the same time External Consultant may also want to seek advice of the employees the two organisations. Organisations' employees and the External Consultant play the role of an End-user. Organisations contact an IESP Administrator that provides communication service, through a Communication Service Provider (CSP), (which is another organisation, playing role of third-party SP). There are two more "management" actors in this scenario: Organisation Accounting Management, who receives and pays bills on the behalf of these organisations; and an External Consultant Service Centre, who deals with the bills that are sent to External Consultant. The IESP Administrator does the job of charging and billing of service usage and settles the accounts among. B2B cooperation takes place between Organisations, External Consultant, and IESP.

\subsection{Initial Accounting Management Information Model}

Elements of service usage information that federated accounting management processes (i.e., service mediation, rating, and billing) use are modelled on the IPDR XML Schema and schema instances (or IPDR documents) are used to produces charges and bills. Developer can extend the elements of IPDR XML Schema for service-specific charging and billing. For the validation of the federated accounting architecture through a trial implementation, VoIP and a media-on-demand service, called MediaShop, are used as two chargeable services. The Figure 5 illustrates the MediaShop service-specific XML schema. A VoIP service-specific schema is being specified.

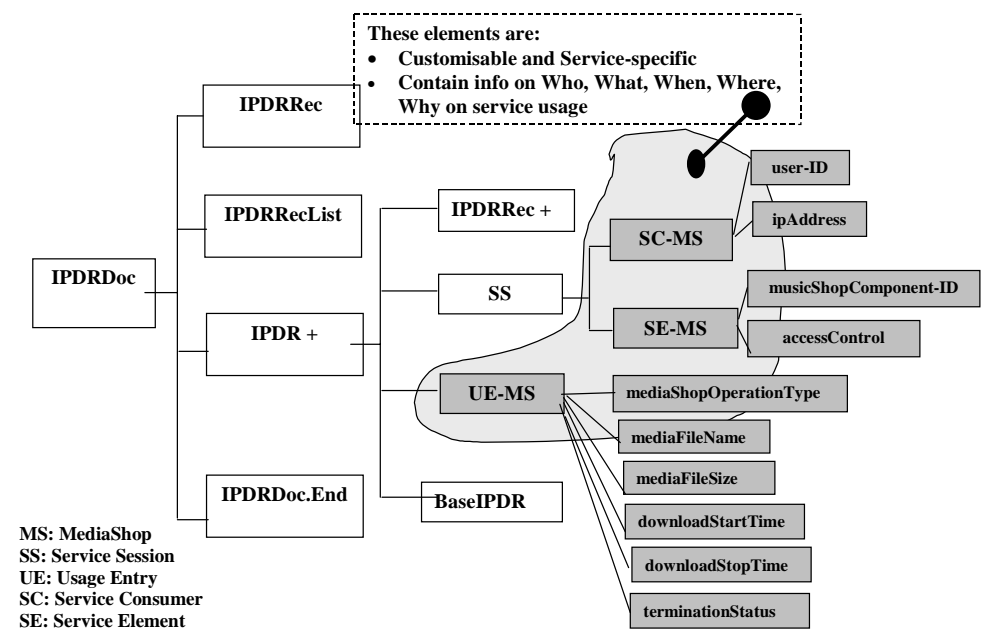

Figure 5: Initial Accounting Management Information Model.

\subsection{Initial Architecture of Federated Accounting Management}

An initial architecture of federated accounting management solution is given the figure 5 and main building blocks of the architecture are described below. 


\section{Customer Accounting Management}

The main emphasis of the work that is reported in this paper is not customer accounting management. However, there are certain aspects of customer accounting management that are addressed. Customer accounting management allows business customers to monitor outsourced service costs on-line. Within the FORM accounting and billing work, active research is being conducted to examine the characteristics of customer accounting management with focus on ease of use, speed of response, minimum "clicks", get the answer right the first time, monitoring per service, monitoring per closed user group. The Customer Accounting Management Agent automates processing of accounting data to be displayed on Customer Accounting Management Console. The Customer Accounting Management Console provides the graphic interface to on-line cost monitoring. It enables the customer to access service accounting information directly from the Accounting Databases Centre. The Accounting Data Centre stores Accounting Records and ChDRs (Charge Detail Records).

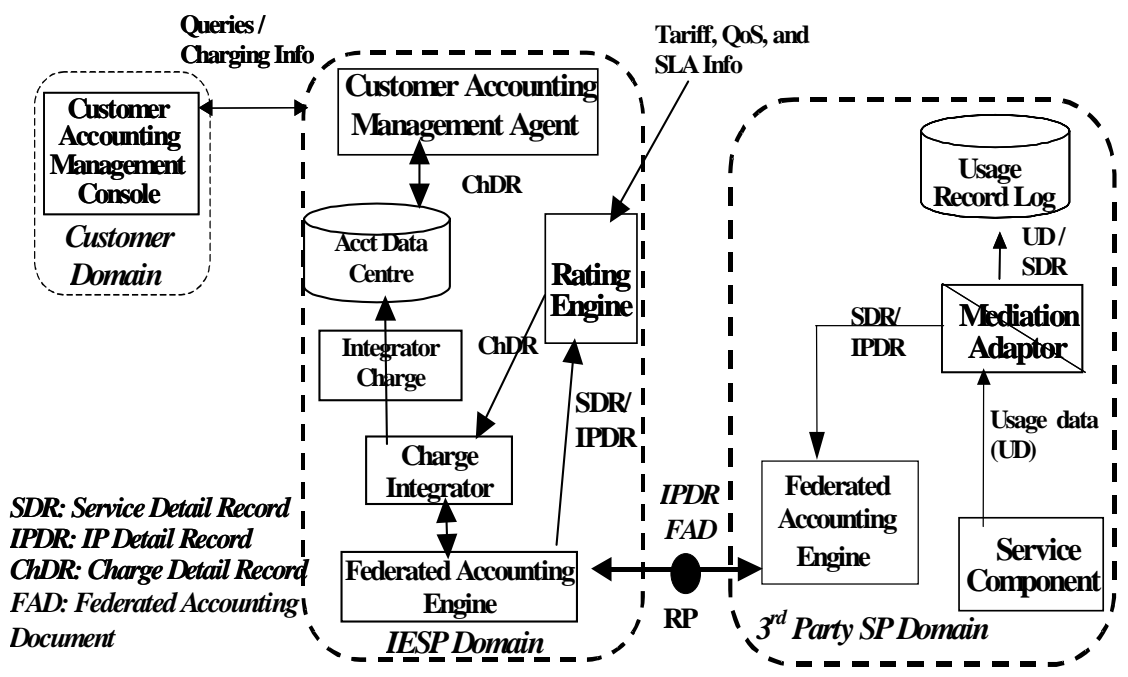

Figure 6: Initial Architecture of Federated Accounting Management System.

\section{Mediator Adaptor}

Its main functions are usage data collection (polling or event-driven) and applying statistical methods (aggregation, correlation, collation). This manages any service usage data collection and provides the rating engine with corresponding SDRs.

This component collects usage data from services (e.g., VoIP or Media-on-Demand) and feeds the data (an standardised record format) into the Rate engine. The records are converted to an format that is suitable as an input to rating. The SDR typically contains the session-id, user id, service-Id and service type, and the actual usage attribute values (usage duration, usage session begin-time and usage session endtime, etc). During a long usage session, this function could be used to gather several intermediate records into intermediate consolidated records. From such consolidated records the total usage can be calculated, which is the sum of the usage of all the intermediate records. 


\section{Rating Engine}

It applies unit charges (different usage measurement units are used for different services), and tariffs (different tariffs are used for different services), on SDRs that it receives from service mediation. Charges are calculated (calculation formula, usage attribute values, etc.) and discounting rules are applied on the basis of SLA.

\section{Federated Accounting Engine}

The Federated Accounting Engine facilitates inter-domain accounting between an IESP and two or more third-party SPs. In order to calculate the charges for end-toend service usage that takes place in the domains of two or more autonomous SPs, accounting information in form of FAD (Federated Accounting Document) is exchanged (see Figure 7 below). In order to meet the requirement of federated accounting, authors have extended IPDR XML Schema by adding $C E$ (Charge Entry) to current IPDR Schema V.2 and used the extended Schema for the representation of usage data, charge, and SPs specific information. The Federation Accounting Engine distinguishes service usage information that is produced within one domain from the usage information that is produced in another domain. It routes the usage information to the IESP accounting system that wants to calculate charges from the usage.

As mentioned in the subsection 3.3 (Dynamic Functionality Requirement), accounting management in a federated environment requires a record format that is agreed upon and exchangeable between IESP and third-party SPs. In the absence of such record format, IESP needs to translate service usage information structured in several different formats (of third-party SPs) into its own record format. Then the usage record can be used to feed the charging and billing processes and to produce a consolidated bill. As the work presented in this paper is currently under development, Figure 7 presents an initial structure and set of information that can be used as a FAD:

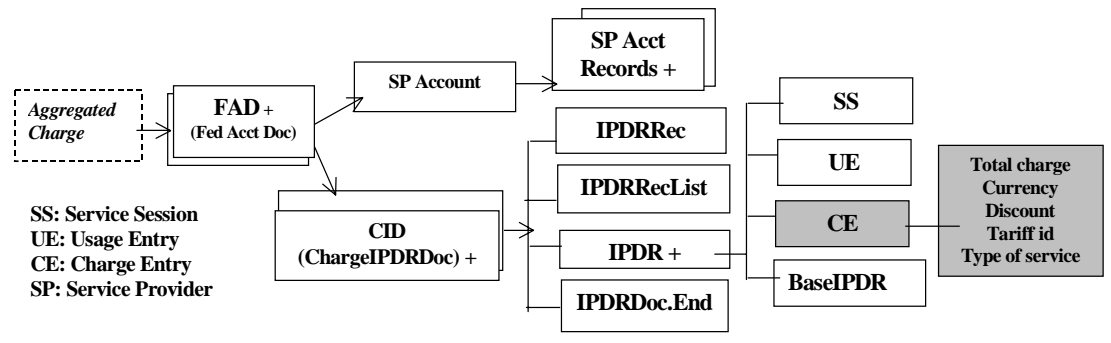

Figure 7: Federated Accounting Document.

\section{Conclusion and Results}

The research work described in this paper aims to develop and evaluate a federated accounting management architecture to support charging and billing of application, communication, and information services. The fundamental idea of federated accounting is based on provisioning, management, and outsourcing of services in a business-to-business environment (also termed InterEnterprise Service, or IES in this 
paper). The underlying theme in this paper is that a single forum cannot address key aspects of federated accounting in a multiple SP environment. Works (e.g., architecture, specification, guidelines) from TMForum, IETF, ETSI, and IPDR are relevant to federated accounting. Therefore, relevant charging and billing concepts from these standardisation bodies should be drawn and converged in a coherent, open and adaptable architectural framework. The initial plan is to evaluate the architecture by developing a prototype of the following accounting and billing management services:

- Charging and billing management in a multi-SP environment;

- Management service for differentiated, usage-based, application-specific charging and billing;

- Management solution for equitable cost allocation and revenue (for sharing accounting information, generating revenues).

The research work has so far developed a business model, a trial scenario, main use cases, and a preliminary information model.

\section{Acknowledgment}

The authors would like to thank David Lewis (UCL London), Søren VejgaardNielsen (now UHC Denmark), and Herve Karp (Atos Integration France) for their comments on federated accounting ideas. The research work described in this paper is being carried out under the auspices of EU-sponsored IST project FORM.

\section{References}

[1] Billing for Next Generation Services: Meeting the ChallengeAn Ovum Report by Keith Brody, David James and Kate Ellis, August 2000.

[2] Business-to-business e-commerce market poised for rapid growth, E-Commerce newsletters, Sell It!, February 17th 1998. http://sellitontheweb.com

[3] Ovum Forecasts the Internet and e-commerce, An Ovum Report by Dr Richard Kee, Dr Henning Dransfeld and Roger Walton, July 2000.

[4] Introduction to Accounting Management, Bernard Aboba and Jari Arkko, IETF Network Working Group, August 1998. Internet Draft: http://www.ietf.org/

[5] Dr Mathew Lucas, "The IP Detailed Record Initiative", Billing World, July/August 1999.

[6] Generic Requirements For Telecommunications Management Building Blocks, (GB909 Part 1) Part 1 of the Technology Integration Map (TIM), Member Evaluation Version 2, TeleManagementForum, March 2000.

[7] Telecom Operation Map, Evaluation Version 2.0, TeleManagementForum, November 1999.

[8] Introduction to Accounting Management, Internet-Draft, Authors: Aboba, Arkko, Harrington, 02 May 2000, <draft-ietf-aaa-acct-00.txt>.

[9] Accounting Attributes and Record Format, Internet-Draft, Authors: Nevil Brownlee, Alan Blount, 14 April 2000, <draft-ietf-aaa-accounting-attributes-03.txt>.

[10] Network Data Management - Usage (NDM-U): For IP-based Service, Version 2, October 2000, IPDR Organisation (www.ipdr.org). 
[11] Internet Protocol (IP) based networks: Parameters and mechanisms for charging, Version 1.1.1, Technical Report, ETSI, 1999-09.

[12] Telecommunications and Internet Protocol Harmonization Over Networks (TIPHON): Inter-domain pricing, authorization, and usage exchange, Version 1.4.2, Technical Specification, ETSI, 1998-12.

[13] "Flexible Multi-Service Telecommunications Accounitng System", Proceedings of INC 2000 (International Network Conference), plymouth, July 2000.

[14] Deliverable D3: System Specification and Models, December 1998, ACTS Project AC335 FlowThru. http://www.cs.ucl.ac.uk/research/flowthru/. 